\title{
Managing Evolution and Change in Web-based Teaching and Learning Environments
}

\author{
Claus Pahl ${ }^{*}$ \\ School of Computer Applications, Dublin City University, Dublin 9, Ireland
}

\begin{abstract}
The state of the art in information technology and educational technologies is evolving constantly. Courses taught are subject to constant change from organisational and subject-specific reasons. Evolution and change affect educators and developers of computer-based teaching and learning environments alike both often being unprepared to respond effectively. A large number of educational systems are designed and developed without change and evolution in mind. We will present our approach to the design and maintenance of these systems in rapidly evolving environments and illustrate the consequences of evolution and change for these systems and for the educators and developers responsible for their implementation and deployment. We discuss various factors of change, illustrated by a Web-based virtual course, with the objective of raising an awareness of this issue of evolution and change in computer-supported teaching and learning environments. This discussion leads towards the establishment of a development and management framework for teaching and learning systems.
\end{abstract}

Keywords: architectures for educational technology systems, distance education and telelearning, interactive learning environments, multimedia/hypermedia systems.

\section{Introduction}

Computer-based instruction is a field that is characterised by fast and substantial developments in technology and pedagogy. Combined with changes required by the organisational environment and inevitable changes to the content itself, teaching and learning environments become increasingly difficult to run. Educators and developers involved in running these systems have become change agents, (Ely, 1999), reacting on external developments and changes. A significant number of systems today explore new technologies for teaching and learning, or evaluate the feasibility of new approaches. Management aspects have often been neglected in the design and development of these systems. The technology is still very young and most of the systems are only in use a few years. This is only slowly becoming a problem, but as we will explain, changes of any kind can have quite severe effects on the system and people involved. As a consequence immense effort and costs are required to maintain the systems and to extend to new developments. A lack of standardised concepts and procedures for design and management contributes to the dilemma. The only solution is to embrace evolution and change from the outset in the design of these systems. As Wilson (1999) says, "the need to adapt to change is already upon us".

Some progress in educational systems development has been made concerning aspects of the product such as how to represent knowledge for Web-based education, or how to store and deliver educational content. The process of development and management has been less well investigated. Authoring of courseware and instructional design has been addressed. However, in particular later stages of this process dealing with management, change, and evolution need more attention. This is particularly important since change and evolution do not only affect the technological aspects. Technology and pedagogy evolve hand in hand. Technology and pedagogy are the two aspects that the two main groups - the developers and the educators - involved in the creation, delivery and management of teaching and learning environments are most concerned with. The overall process of 'engineering' teaching and learning environments concerns, and should involve, both groups. Domain-specific engineering techniques and methods have developed for various areas - examples are hypertext systems (see (Lowe \& Hall, 1999), or (Lennon, 1997)) or eCommerce systems (see (Langer, 2001) or (Treese \& Stewart, 1998)) - usually a few years after the

* Corresponding author. Fax: ++353 +1 700 5442. E-mail address: cpahl@compapp.dcu.ie 
emergence of a new technology. Respective development and management principles are now also sought for teaching and learning environments. We will explore the background for such a framework of change and evolution management.

Our objectives are to create an awareness of the practical aspects of the evolution and change problem - technology, pedagogy and cost related - and to illustrate the problems. Reported experience in managing educational technology over a longer period of time hardly exist; rare examples are (Trikic, 2001) and (Palmer \& Tulloch, 2001). We want to bring factors of change into mind that are sometimes forgotten, ignored or neglected. A structured account of possible factors of change shall be given, leading towards a taxonomy of change factors. We also outline principles of design methodology for change. We will illustrate the evolution of educational systems using the experience with our own virtual Web-based and Web-delivered course as an example. This course system - a second year undergraduate course - has been taught since the early 1990s supported by a hypermedia system and since 1996 as a virtual Web-based course. The system has changed in various aspects over the years. Even though we use a Web-based virtual undergraduate course system as the example, our results apply to a wider range of teaching and learning environments (TLE) - not limited to the Web as the representation and delivery medium, virtual delivery as the mode, or third-level institutions as the target organisation. However, we focus on Web-based delivery and management aspects, excluding authoring and evaluation support. We also limit ourselves to courseware, ignoring aspects of larger environments such as virtual universities and student administration frameworks.

\section{Teaching and Learning Environments}

The notion of teaching and learning environments (TLEs) covers a wide range of computer-based and computer-supported educational systems. In general, a TLE can be described in four dimensions providing different perspectives - graphically summarised in Figure 1:

- Content the subject-oriented perspective - refers to the subject taught and the representation of knowledge in the TLE.

- Format the organisational perspective - comprises attributes determined by the institutional context: curriculum, syllabus, staffing, etc.

- Infrastructure the technical perspective - relates to the hardware and software environment in which the TLE is deployed.

- Pedagogy the educational perspective - refers to the instructional design of the TLE determining in which way the course is taught.

TLEs can be described in terms of attributes along these dimensions. We identify some attributes in particular of the pedagogy dimension - which characterise TLEs that are particularly affected by change: multiservice, multimedia, interactive, autonomous, adaptive, and collaborative TLEs. In multiservice environments, different services - supporting different learning activities such as attending a lecture, participating in a guided tutorial, or practising in a lab - are integrated within one system. A multimedia environment uses various media, ranging from text to audio and video. In our own course we have used a synchronised audio/visual presentation for lectures. Learning by doing - supporting active learning - is the approach that is supported by interactive environments. Meant by this are features of the system that the student can interact with in order to learn or train a specific topic or skill. In autonomous environments, the system replaces major functions of the educator. This includes elements such as assessment, learner dialogue and feedback. Adaptive systems personalise the delivery of the system; the system adapts itself to a particular student's characteristics and needs. Finally, in collaborative systems, the communication and co-operation between students is supported - typically by offering both synchronous and asynchronous means of communication such as email, chat, newsgroups, but also shared workspaces to develop coursework in groups. Systems characterised by these attributes typically need strong technological and administrative support in order to facilitate these features. Changes affecting these systems often result in extensive re-implementations. These systems become managed learning environments, with educators and developers in charge of the management.

There is a close relationship between the pedagogical concepts supported and the enabling technology. Interactive services support the active learning paradigm; communication services such as email, chat, or shared spaces support collaborative, group-oriented and distributed learning; multimedia technology creates an engaging learning environment; a multiservice environment supports multimodal learning, etc. The development and evolution of technology is crucial because of two reasons. Firstly, 
changing technology impacts the IT infrastructure of the TLE. Secondly - and more interestingly -, new hardware and software technologies enable new pedagogical approaches to be implemented. We distinguish technology infrastructure and feature-specific technologies, called vertical and horizontal technologies relating to the architecture of a TLE. This classification will help us later in structuring and assessing the impact of factors of change. Vertical technologies are infrastructure technologies that comprise basic functionality. For instance, Web technology, networking and communication, and storage facilities are infrastructure technologies for Web-based courses. Horizontal technologies are featurespecific technologies that enable certain forms of learning. An example is active learning, which is supported by interactive and streaming media. Another way of classifying technologies is to distinguish common and education support technologies. Common technologies shall be those technologies that are available in most computing environments and that are not specific to educational purposes. We look at common technologies only, ignoring education-specific support platforms such as systems like WebCT, FirstClass or Blackboard, see (Anderson \& Jackson, 2000) for an overview. Our remarks certainly apply to the latter as well, since they also exhibit some of the crucial attributes.

\section{A Virtual Database Course}

Our own course - an introduction to database systems - shall be introduced from a historical perspective, showing its evolution over the years. This course is an undergraduate course for a computing degree, taught as a virtual course. Our course is Web-based, i.e. uses an open standard as the basic platform. This guarantees the usability of the course without the need to install any other software at the student's side except a Web browser with standard plug-ins. The history of this system reaches back to the early 1990s when a hypermedia system was developed, (Smeaton, 1991). The course system has been reengineered in 1996 as a multimedia lecturing system for the Web, (Smeaton \& Crimmins, 1997) or (Smeaton \& Keogh, 1999). The lecture service transforms the classical idea of lectures - a lecturer speaking to students supported visually by a blackboard or overhead projector - into the virtual world. An audio stream capturing the lecturer's speech is synchronised with the presentation of visuals.

In 1999, this system has entered the third major development phase, (Pahl, 2001a) and (Pahl, 2001b). It has been extended to an integrated multiservice environment. New interactive features, providing interactive tutorial and lab services, have been integrated into the system. The course now supports several learning modes - attending lectures, tutorials and labs - through an integration of different educational services. Interactivity is a crucial element in a virtual course, since it allows us to engage the student. The interactive part of our virtual database course provides several lessons arranged as a guided tour through the database language SQL. The student can submit solutions to small problems to a remote database server, which processes the student input and returns a result. The lectures including the audio material is integrated with the interactive services so that look-up of material is easy. Self-assessment functionality is provided in order to make the learning experience more successful.

Another development is currently in progress, (Pahl, 2001c), in that we have re-engineered the HTML-based course content in a more flexible XML-representation - (W3C, 2001). The content and also the learner data is now stored in a database, which allows the configuration of content based on specific needs, for example the needs of a particular student. Flexible representation of material is the key to adaptive and personalised systems and having content marked up in XML allows us to explore this feature further.

An essential question concerns the transferability of our results. To a certain extend this means how prototypical or representative our system is. The key features of our system are also found in a variety of other systems - systems with interactive elements, multimedia features, or flexible content representation. Various other systems use multimedia technology such as audio and video material, e.g. (Bouras, Hornig, Triantafillou \& Tsiatsos, 2001). Klett (2001) reports about a system with interactive features. Inoue and Ueno (2001) describe multimodal learning supported by several different educational services. Flexible representation of course material using XML is also used by Bielikova (2001) and Quentin-Baxter \& McDonald (2001). Even though the focus in those publications is rarely on change and evolution, similar problems are likely to be encountered in a long-term use of these systems.

\section{Factors of Change}

Currently, a general trend in educational technology can be observed that indicates a shift from teaching to learning according to Okamoto, Christea \& Kayama (2001). The student in these environments 
becomes more independent from the teacher. New technologies allow new forms of educational support to be facilitated, enabling new pedagogical concepts, (O’Donoghue, Singh \& Dorward, 2001). As a consequence, the role of the teacher changes from an educator to a facilitator and manager. However, these developments are only part of the changes that impact TLEs. Besides the evolution of technology and pedagogy, the changes inflicted by the day-to-day business of an organisation or the evolution of the subject domain need to be considered.

Our virtual course has evolved in several major phases focussing on hypertext representation, the Web and multimedia, interactivity, and adaptivity, respectively. However, the system had been subject to change constantly in the periods between the major development phases. The factors that influence the implementation of teaching and learning environments, and that require changes are numerous. Essentially, we can distinguish four main classes of factors of change - which we structure along the dimensions of TLEs:

- Content: Changes relating to the subject that is taught.

- Format: Changes related to staff and students involved or to curriculum and syllabus.

- Infrastructure: Changes due to developments in hardware/software technology or learning devices.

- Pedagogy: Evolution of teaching and learning in computer-supported environments.

The IT infrastructure and pedagogy evolution is a general process affecting all, whereas evolution in content and format is specific to an individual course or to an organisation. However, all aspects can have a severe impact on the system in use. We shall now discuss these different classes separately. We identify different single factors in these categories and illustrate them using our own course.

\subsection{Content}

Both external and internal factors, i.e. factors that are without or within control of the educator, can result in content changes:

\section{- Subject evolution: The course subject itself evolves - an external factor.}

- Content improvement: Content is changed in order to improve the material in a planned process.

The evolution of the course subject is an external factor. Evolution in the subject area can force the course material to be updated or redeveloped. An example for our database course is the emergence of Web databases and the need to cover this topic from a theoretical as well as practical point of view with additional lectures, but also with adequate interactive tutorial and lab material.

There can also be change factors internal to the course, i.e. factors planned to be involved in the design. Planned content changes are based on evaluation results that are used to improve the course itself.

Content changes and updates are usually easy to carry out - at least as long as text is the medium. For audio and video material more technical preparation is needed. For example, for audio recordings a problem can arise if the previous speaker is not available any more.

\subsection{Format}

The organisation, which offers and runs a particular course, can require changes in a variety of ways that affect the format in which the course is taught. Factors of change arising from the institutional context in which the course is provided are the following:

- Staff: Changes relating to educators, course developers, or technical support staff.

- Students: The student body changes in terms of numbers, qualifications, or mode of learning.

- Timetabling: Changes related to where and when a course takes place.

- Syllabus: Content and organisation of the course content can change.

- Curriculum: Organisational needs require changes in level, extent, or prerequisites of courses.

- Environment: Issues such as the legal or financial environment require changes.

Significant disruptions in the delivery are usually caused by staff changes - this includes educators, course developers and technical support staff - in particular when a course environment is tailored towards interests and teaching style of the previous teacher in charge. Our own system has been taught by two lecturers, one teaching the course until 1999, and the other since then. These two periods coincide with the essential phases of the course development - the hypermedia and multimedia lecture development until 1999 and the interactive and adaptive services development since then. Both developments were driven by the interest of the lecturers in the respective features. In both cases the lecturers were in charge of the development of the features, supported by research assistants and programmers. A crucial issue is that most of the developers involved in the actual implementation of our system have left our university in the 
meantime. Specific knowledge and expertise is no longer available - an issue that has been painfully experienced while relocating the course system from one hardware platform to another.

Over the years, the student body usually changes in a number of ways: total numbers, qualifications, or mode of learning, e.g. full-time vs. part-time or distance vs. local attendance. The introduction of parttime or distance schemes certainly causes major changes in a system. A scalability problem occurred when our student intake for the course was doubled. The database system originally used to support the interactive elements provided a too limited number of possible connections and showed to be unstable under high loads. A larger database system replacing the previous one had to be installed as a consequence of this technical insufficiency.

Times and places - the timetabling of the course - is relevant for hybrid courses or for distance delivery where online meetings and discussions have to be scheduled. These might affect the form of presentation. For example, circumstances decide whether synchronous or asynchronous discussions are held for a distance education course.

The syllabus might have to change from time to time - this can be a consequence of changes in the subject area itself or changes in the curriculum, but sometimes there could also be an independent decision to focus more on for instance practical elements without another external cause.

Another factor concerns issues arising from the context in which a particular course is embedded the curriculum. Due to organisational needs a course might change with respect to its level, extent, or the pre-requisites. This is partly reflected by the student change factor. Our course has been moved to another year in the computing degree program, and a variant has been offered to a different degree programme with different credits or measurements of workload. In general, this implies that the course content might need to be reduced or extended.

In a wider sense, we can also include changes in the legal environment. An open question in various institutions and countries addresses intellectual property rights. What, for instance, happens if a course developer leaves an institution? Costing is another related element, which needs to be considered. An institution might decide because of financial reasons to discontinue the support for a particular technology.

\subsection{Infrastructure}

Developments in information and communications technology certainly influence the infrastructure and architecture of educational systems. We can distinguish these technological factors into computer hardware technology, systems and language technology, and learning devices:

- Hardware technology:

Communications and network technology, computing power, and computer platform have constantly improved.

- Systems and language technology: Minor changes within the technology, technology leaps, legacy or pre-eminent technologies are frequent issues.

- Learning devices: Software and hardware devices such as smart objects, information infrastructures and virtual environments serve as learning devices.

Changes in hardware technology comprise communications technology and computer technology. Communications technology relates essentially to networks. An example is the increase of bandwidth in the past, allowing more data to be transferred in shorter time. In particular upgraded modem technology on PCs owned by students has improved the delivery and has made for example streaming of our audio material to PCs at home feasible. Improvements in computer technology have led to computing power increases and a substantial decrease in prices for PCs. Both factors have positively influenced PC ownership among students. More processor power and greater bandwidth are beneficial, if for example computers in labs are replaced. However, sometimes these replacements can introduce a new hardware platform - with possible portability problems for TLEs running on the previous platform.

Systems and language technology relates to computer software and languages. We can distinguish minor changes - essentially caused by versioning of products and languages, possibly requiring small updates in the TLE and major technological leaps. Occasionally, technology leaps occur where new technologies are introduced, sometimes even requiring existing ones to be replaced. An example for a technology leap is the emergence of the World Wide Web in the early and mid 1990s. A number of systems, such as ours, have existed before that. The phenomenal success and proliferation of the technology made the discontinuation of the previous technology mandatory. This has resulted in a substantial redevelopment since both the content representation and the delivery mechanism had to be changed. 
Sometimes, the introduction of new technologies cannot be ignored, in particular when an existing technology becomes legacy and required support ceases to exist. A related problem occurred in our course when a data format for audio material, used in 1996 to record lectures and being the standard at that time, was replaced in 2000 by another, more advanced format as the standard. Using the 1996 format with the most recent versions of the corresponding audio player requires an additional module to be loaded and installed for the player. In the basic version of the player, the support for the old format is discontinued. Another example of technologies requiring TLEs to be changed is the pre-eminence of new technologies. XML - the eXtensible Markup Language - is a highly acclaimed technology, and has certainly impacted educational systems development. A variety of newer systems use XML as the main content representation format, which has the property of being an interoperable data interchange format. Interfacing with these systems, or attempting to meet standards formulated in XML then requires the conversion to XML. A further example of a new technology that has been introduced into the educational context are metadata frameworks describing educational objects in abstract terms, allowing them to be discovered and compared. An XML-based version of such as framework is the IMS metadata standard for teaching and learning objects. A technological framework for metadata is RDF, the Resource Description Format.

Learning devices are certainly part of the technology aspect. However, we separate hardware and software technology from devices, which can be packages of hardware and software. The learning device in our case is the Web browser - a software device. Evolution in hardware and software technology can make other forms of devices possible, see (Dede, 1995b). New hardware and communications technologies can lead to smart objects using mobile microprocessors and wireless communication - devices that can be integrated into learning environments. Recent advances in handheld and PDA technologies are examples. Information infrastructures provide remote access to experts, archival resources, etc. Knowledge Webs are an example of this kind. The re-engineering of an existing system to a new platform also falls into this category. A typical example is the conversion of CD-ROM based courses to the Web. Shared virtual environments range from single-user simulators to group- and community-oriented virtual worlds. The conclusion that has to be drawn from the evolution of learning devices is to design courseware with open, flexible, and interoperable architectures in mind.

\subsection{Pedagogy}

The state of the art in instructional design for computer-assisted teaching and learning has been strongly influenced by advances in information technology, education and cognitive sciences. In particular for Web-based systems the aspects knowledge modelling, active learning, personalised learning and collaborative learning have been investigated intensively.

- Knowledge modelling: $\quad$ Acquisition, modelling of and access to educational knowledge.

- Active learning:

- Collaborative learning: Engaging the student through interactive systems.

Supporting communication and collaboration through communications systems.

- Autonomous learning: Personalisation and independent learning through adaptive systems.

- Evolving instructional design: Planned evolution integrated in design through course evaluation.

The first four elements describe the stages that the development of educational technology went through over the last years.

Knowledge modelling is a central activity. The evolution of computer-supported TLEs has started with systems providing easy access to course material in a different form. An issue that seem to appear again as a research topic is the organisation of learning resources. In particular in personalised and adaptive system, the representation of learning material has to be co-ordinated with the learner model. A wider approach than structured representation of knowledge is required. Web technologies have improved access significantly, compared to the various forms of hypertext and hypermedia systems that existed before. Web-enabled databases allow the flexible storage and access to course content and learner data. In our particular case, we have re-engineered the original HTML-material into XML and provided flexible access for configuration and delivery through database storage.

The development of advanced Internet and Web technologies such as mobile code has enabled interactive elements - students can respond to the presentation of material and proactively approach a system. The paradigm of active learning can be supported. The availability and accessibility of various forms of media - such as audio, video, and virtual worlds - supported by the Web has led to an 
improvement of interaction and simulation features. We have used advanced HTML- and servertechnology to implement our interactive services

Collaborative learning systems use communications technologies ranging from email, chat rooms and news groups to video conferencing systems - essential for distance education and distributed learning. Collaborative, group-oriented learning is facilitated. Our course is taught on-campus. Therefore, direct contact between teacher and students, and among students, is possible.

Personalised adaptive systems have been introduced based on new server and database technologies, allowing developers to store user information and create content dynamically. Independent learning is supported by technologies based on personalisation that provide self-assessment and feedback functionality. Autonomous learning allows the student to take control over her/his own organisation of learning. Our course system implements some scaffolding features, i.e. support for the independent learner in form of feedback, self-assessment and links.

Evolution through evaluation is an iterative, incremental approach to improve content and instructional design. Essential for any TLE is the constant monitoring of students and their progress and the evaluation of the course and the system effectiveness. We have used evaluations also to detect errors and inconsistencies in the content itself, but mainly to improve the instructional design.

The role of technology evolution as an enabler of new pedagogical concepts is evident. However, student usage of computer-supported systems is far from being well understood and, consequently, the evolution of technology and pedagogy for these systems will continue for some time before agreed understanding and standards will have emerged.

\subsection{Technology and Pedagogy Evolution}

The close relationship between pedagogy and technology evolution is an important aspect in designing and managing educational systems. Often, systems will undergo major redevelopment to support new forms of instructional design supported by new technological developments - as it has happened twice with our system. Some of these associations between technology and pedagogy are multimodal learning as the pedagogical approach and educational service integration as the enabling technology, or independent learning as the pedagogical approach and scaffolding - the provision of links, help, self-assessment etc. as the enabling technology. Understanding this relationship is crucial for the anticipation of change and the most effective reaction to it.

Overall theories are sought linking pedagogy and technology together. Activity theory is a conceptual framework that can describe the structure, development, and context of computer-supported activities, (Nardi, 1997). Its emphasis on the interaction between agents and their environments explains the principle of tool mediation. Tools shape the way humans interact with reality. Tools reflect experiences other people have made in trying to solve similar problems. This experience is accumulated in structural and behavioural properties of the tool. A TLE is the tool that provides a student with access to a part of the reality - the course subject - guided by structural and behavioural rules defined by the educator. Approaches such as problem-based learning, constructionism, or exploratory learning can be derived from constructivist theory, see (Dede, 1995a). Constructivism says that knowledge is constructed by a student, rather than taught to the student. The process of knowledge construction is viewed as deeper than the traditional approach. The student is engaged in solving meaningful problems - we have called this active learning. Interactive services can enable active learning in a constructivist style if they create a representation of reality in which learning is relevant. Activity theory or constructivism together with technological developments can be the drivers of the evolution of TLEs. Instructional design is the crucial activity that embraces these pedagogical and technological developments. Courses need to be designed using the most appropriate learning approaches for the subject restricted by the technology that is available.

\section{Development and Management for Evolution and Change}

Evolution and change are ubiquitous - see Figure 2 - which can, as we have seen, pose some difficulties for the change agents. Both educators and developers act as change agents for computer-based educational systems. An adequate methodology for the support of change can provide help for the development and management of these systems. Standard methodologies for the development of software often address the problem of change and evolution. However, the context of education requires some consideration. 


\subsection{Context for a Methodology}

A methodology for education engineering - an engineering approach to the systematic and methodological development and management of TLEs - could hold the key to the change problem. Ultimately, we would like to achieve maintainable TLEs, i.e. TLEs that are scalable, configurable and interoperable in order to deal with change. We consider such a framework necessary in order to cope with the consequences of evolution and changes in technology, pedagogy, content, and the institutional context. The presentation of a complete methodology for change is beyond the scope of this investigation. However, we discuss the context and the key aspects.

The characteristics we have listed for TLEs are desirable for any computer system - some authors such as Virvou and Tsiriga (2001) have addressed general software development issues in the educational context. However, it is worth looking more closely at where educational systems differ from computer systems in general. Educational technology is still less mature than classical computer systems technologies such as business applications. The users are not well understood. How students learn with computer-based system - the learning process - needs more research. The goal of learning is specific to the area. Learning is a complex process that can not easily be compared with goals in other computer-based systems - such as shopping at e-Commerce Web sites or administrative tools where the tool carries out tedious work. The main person in charge of developing and running an educational system - the educator - usually does not have significant technological background. Teaching is seen by most as an art and often involves very individualised skills and personal styles. The institutional context is different from classical business and administration software. These differences call for a domain-specific evolutionary methodology for instructional design and implementation.

\subsection{Key Aspects of a Methodology}

A variety of factors can cause a TLE to be changed, see Figure 2. Change is pervasive in these environments. A sustainable approach to the development and management of TLEs embracing change and evolution from the outset is sought. The methodology has to consider the changing roles of educators and developers. Elements of a methodology for incremental design and evolution for TLEs should include the anticipation of change and the consideration of innovative developments, architectural aspects, and standards, see Figure 3. These aspects need to be considered for all stages of the TLE life cycle - such as design, deployment, and evaluation.

An essential lesson we have learned is that change has to be anticipated and reflected in the design. The fact that neither the content of our courses nor their metaattributes such as format, infrastructure and pedagogy are static needs to be accepted. Requirements are volatile - some are mutable, others will only emerge during the development or deployment. We have listed the factors of change in different categories, hoping that these might help to devise designs that allow us to deal with these changes with respect to the different categories. What is sought is an incremental, iterative method for ongoing construction and reconstruction that can cope with the changes that are required.

Another lesson learned is to look at new innovative developments at the verge of making an impact. The design of TLEs - in particular for Web-based environments for distance access - has to aim at the lowest common-denominator technology available in order to make distance access acceptable for students working from home. This gives the chance to consider emerging technologies and their maturation for future changes of the system. A technical horizon of several years can be incorporated. Interoperability is one of these emerging aspects, concerned with integration and interfacing educational systems among each other. Our experience with change and evolution can ideally be projected into the future through an appropriate design for change methodology.

The architecture of a TLE plays an important role in the determination where a change actually takes place. We can relate factors of change to different parts of a TLE architecture. The resources are affected by content factors, the configuration component is affected by format and content factors, and the delivery system is affected by factors of all four dimensions. Although we have not directly used the Learning Technology Standard Architecture (LTSA) defined in (IEEE LTSC, 2001) as our architectural framework, there is a correspondence between the processes in the LTSA and our components ${ }^{1}$. Most often resources

\footnotetext{
${ }^{1}$ Resources are distinguished into learner records and learning resources. The configuration is the essential task of the coach. The delivery service is identified in both architectures. We have neglected the evaluation service. Its position in the architecture would be similar to the delivery service.
} 
are affected by changes. However, due to their static nature, changes to resources - to be carried out by an educator - tend to be simpler than those effecting for example the delivery system where usually implementation work - to be carried out by the a developer - is required. We have divided the technologies into horizontal and vertical technologies. Horizontal technologies - such as interactive multimedia, or collaboration technologies - form an orthogonal dimension to the vertical dimension - resources, configuration and delivery. Educators and developers - the two change agents involved - are affected in different ways. The educator is most affected by changes in pedagogy, content and format, whereas the developer is most affected by changes in pedagogy and infrastructure.

Standards play an important role in the development of any system. We can distinguish de facto standards, possibly dictated by concepts of best practice for the context, and standards presented by professional or standardisation bodies. Examples for the latter are subject-specific technology standards such as those suggested by the IEEE Learning Technology Task Force LTTF, or general education-oriented ones such as the standards and guidelines published by the American Association for Higher Education AAHE or the Association for the Advancement of Computing in Education AACE. Standards such as the IMS metadata framework (IMS, 2001) rely in their implementation on existing technologies - XML in that case. Other frameworks are usually platform-independent, such as the IEEE Learning Technology Standard Architecture LTSA or the Learning Object Metadata standard LOM. Standards affect the pedagogical side as well as the technical side. For the technological side, open source infrastructures and interoperability are key issues in this context. These open source standards, such as Web technologies, determine the interoperability of services with other services or systems. In the future, the need to integrate, to interface and to combine will increase. The use of standards should not be restricted to technologies for the product itself, but should also be applied to the development and management process.

\section{Conclusions}

The design of Web-based courses can be described in terms of the four dimensions content, format, infrastructure and pedagogy. Our classification of change factors along these dimensions shows the ubiquity of change and evolution. We have seen that these factors are manifold and that they can have severe consequences. Change agents in educational systems - the educator and the developer - have to react to change and evolution. Change might be beneficial, such as new pedagogical developments, or might just be forced upon the system through administrative and technical changes in the environment; in any case it comes with a cost from a management point of view. In order to keep costs low and to minimise them whenever possible, the need to plan for change and evolution in instructional design and management is imperative.

Educational technologies lack sufficient support for change and evolution. Let us summarise some of our key observations reflecting the current problems. Firstly, even though some progress with respect to standards has been made - Anido, Llamas, Fernandez, Rodriguez, Caeiro \& Santos (2001) describe some efforts - the standardisation of technology, development and management techniques and procedures is not complete. Secondly, the life expectancy of technologies is usually limited to a few years before new ones dominate. Thirdly, costs have been underestimated in the past. Cost-effectiveness so far could only be achieved for large student numbers. Finally, staffing is often inadequate - the development and deployment of educational systems depends much on the enthusiasm of individuals. In the presence of change and evolution, problems are inevitable. We have demonstrated the consequences - difficulties in the reaction to change and in long-term planning - in our presentation of change factors. Planning with change in mind helps to manage courseware in a rapidly evolving environment cost-efficiently and increases the chance of including innovative developments into educational systems. System requirements are volatile and subject to change. As a consequence, requirements should embrace change and should reflect the possibility for change explicitly.

We have suggested some changes to the development and management of educational systems in order to deal with the difficulties we just described. Courses in the future need to be much less an expression of the educator's style of teaching. The anticipation of staffing changes makes this necessary. New standards are another issue - current efforts need to be closely observed and incorporated into designs. Current and future technological innovation will enable new pedagogical concepts for TLEs. Pedagogical needs and desired features and the development of technology go often hand in hand. The future seems to show two developments that can impact computer-supported educational systems. Firstly, an increased 
mobility of people, devices and computer programs, and secondly, the evolution of the Web from an information-oriented to a service-oriented framework.

One lesson that we have learned - supported by other authors such as Palmer and Tulloch (2001) concerns a more strategic level. High-end features in educational systems are showpieces that raise the profile of the developer. However, these systems have turned out to be costly and difficult to maintain. New investments and new developments are required if new concepts are to be implemented. The development of easy-to-use infrastructures and mainstream systems should be favoured if maintainability and not the exploration of new technologies is the objective. Even in the latter case, the technologies might remain in service for several years and, consequently, need to be maintained as well. Palmer and Tulloch point out that the implementation of high-end technology usually comes at a price and such developments are only sustainable if there is sufficient backing from the organisation in charge.

Our conclusion is that a development and management methodology for TLEs - a framework of education engineering - is needed. Wilson (1999) has pointed out that conceptual frameworks for learning support are always struggling to keep up with constantly developing technologies and theoretical advances in education and cognitive sciences. In order to keep TLEs manageable and cost-efficient, we propose a systematic evolutionary approach to the development and deployment of these environments, acknowledging the different roles of the change agents. The anticipation of change and evolution needs to be incorporated, and all factors of change have to be understood and considered. Standards are of paramount importance. Such a framework extends to the technology in use, but also to the representation and organisation of course content. Our objective has been to raise an awareness of the evolution and change problem, and to present the foundations of a methodology. Educators and developers need to be aware of changes in their environment and also changes to their own role, and in particular, need to adapt to change and evolution by planning for the management of change already in the design.

\section{Acknowledgements}

The author is greatly indebted to Alan Smeaton, who started developing the system described, and who also commented on a draft version of this paper.

\section{References}

Anderson, M., \& Jackson, D. (2000). Computer systems for distributed and distance learning. Journal of Computer Assisted Learning, 16, 213-228.

Anido, L., Llamas, M., Fernandez, M.J., Rodriguez, J., Caeiro, M., \& Santos, J. (2001). A Standards-driven Open Architecture for Learning Systems. Proc. IEEE International Conference on Advanced Learning Technologies ICALT'01, 3-4.

Bielikova, M. (2001). Adaptive Presentation of Evolving Information Using XML. Proc. IEEE International Conference on Advanced Learning Technologies ICALT'01, 193-196.

Bouras, C., Hornig, G., Triantafillou, V., \& Tsiatsos, T. (2001). Architectures supporting e-Learning through Collaborative Virtual Environments: The case of INVITE. Proc. IEEE International Conference on Advanced Learning Technologies ICALT'01, 13-16.

Dede, C. (1995a). The evolution of constructivist learning environments: Immersion in distributed, virtual worlds. Educational Technology, 35(5), 46-52.

Dede, C. (1995b). The Evolution of Learning Devices. http://www.ed.gov/Technology/Futures/dede.html.

Ely, D.P. (1999). Conditions that Facilitate the Implementation of Educational Technology Innovation. Educational Technology, 39(6), 23-27.

IEEE Learning Technology Standards Committee LTSC (2001). IEEE P1484.1/D8. Draft Standard for Learning Technology - Learning Technology Systems Architecture (LTSA), 04/06/2001. IEEE Computer Society

IMS Global Learning Consortium (2001). IMS Web Page http://www.imsproject.org.

Inoue, T., \& Ueno, H. (2001). NACSIS-ILL WWW Based Training System. Proc. IEEE Symposium on Applications and the Internet SAINT 2001 - Workshop on Internet-Supported Education, 39-44.

Klett, F. (2001). A Design Framework for Interaction in 3D Real-time Learning Environments. Proc. IEEE International Conference on Advanced Learning Technologies ICALT'01, 63-66.

Langer, A.M. (2001). Applied E-commerce: Analysis and Engineering for E-commerce Systems. John Wiley \& Sons

Lennon, J.A. (1997). Hypermedia Systems and Applications. Springer-Verlag. 
Lowe, D., \& Hall, W. (1999). Hypermedia \& and the Web - an Engineering Approach. John Wiley \& Sons.

Nardi, B. (Eds) (1997). Educational Context and Consciousness: Activity Theory and Human-Computer Interaction. MIT Press.

O’Donoghue, J., Singh, G., \& Dorward, L. (2001). Virtual education in universities: a technological imperative. British Journal of Educational Technology, 32(5), 511-523.

Okamoto, T., Christea, A., \& Kayama, M. (2001). Future integrated learning environments with multimedia. Journal of Computer Assisted Learning, 17, 4-12.

Pahl, C. (2001a). Interactivity and Integration in Virtual Courses. Proc. International Conference on Advanced Learning Technologies ICALT 2001, 395-396.

Pahl, C. (2001b). The Evaluation of Educational Service Integration in Integrated Virtual Courses. Proc. Symposium on Applications and the Internet SAINT'01 - Workshop on Internet-supported Education WISEd'2001, 61-66.

Pahl, C. (2001c). XML-Technologies for the Support of Active Learning in Interoperable and Open Webbased Learning Environments. Proc. $6^{\text {th }}$ WebNet 2001 World Conference on the WWW and Internet.

Palmer, S., \& Tulloch, W. (2001). The Evolution of Online Teaching and Learning in Engineering at Deakin University. Journal of Computing in Higher Education, 14 (1).

Quentin-Baxter, M., \& McDonald, T. (2001). Managing networked learning environments for health via XML databases and Zope. Poster Proc. $10^{\text {th }}$ International World-Wide Web Conference, 114-115.

Smeaton, A.F., \& Crimmins, F. (1997). Virtual Lectures for Undergraduate Teaching. Proc. ED-MEDIA'97 World Conference on Educational Multimedia and Hypermedia.

Smeaton, A.F., \& Keogh, G. (1999). An Analysis of the Use of Virtual Delivery of Undergraduate Lectures. Computers \& Education, 32(1), 83-94.

Smeaton, A.F. (1991). Using Hypertext for Computer-Based Learning, Computers and Education, 17(3), 173-179.

Treese, G.W., \& Stewart, L.C. (1998). Designing Systems for Internet Commerce. Addison Wesley.

Trikic, A. (2001). Evolving open learning environments using hypermedia technology. Journal of Computer Assisted Learning, 17, 186-199.

Virvou, M., \& Tsiriga, V. (2001). An object-oriented software life cycle of an intelligent tutoring system. Journal of Computer Assistd Learning, 17, 200-205.

Wilson, B.G. (1999). Evolution of Learning Technologies. Form Instructional Design to Performance Support to Network Systems. Educational Technology, 39(2), 32-35.

World Wide Web Consortium - W3C (2001). The Extensible Markup Language (XML). Web page http://www.w3.org/XML/. 


\section{Captions}

Figure 1. Dimensions of Teaching and Learning Environments.

Figure 2. Dimensions of Change Factors.

Figure 3. Aspects of a Methodology for Change. 

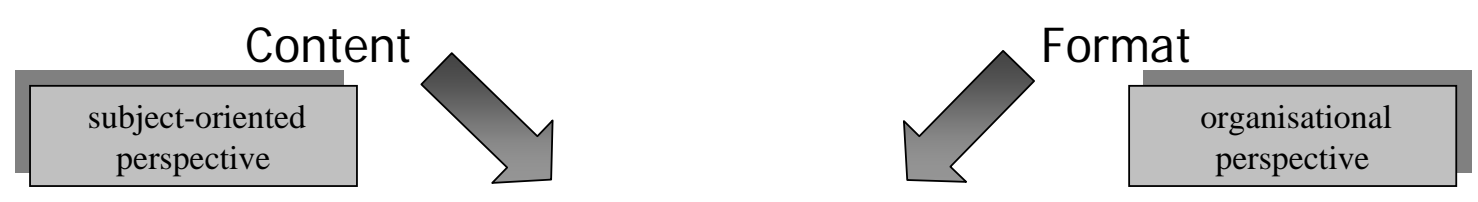

\section{Teaching and Learning \\ Environment}

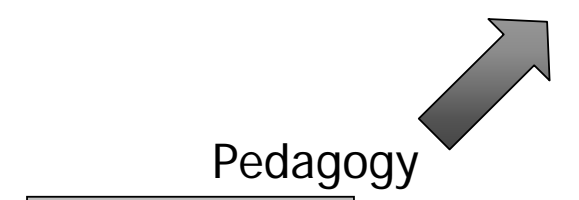

educational

perspective
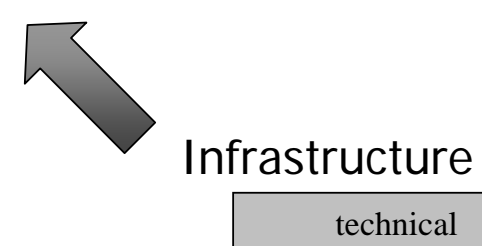

perspective 







\section{Factors of Change}

- Dimensions of Change

- Anticipation of Change

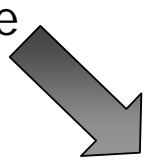

Methodology for Change

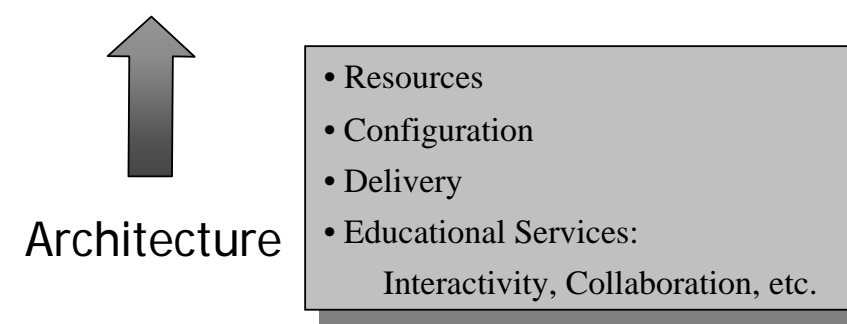

